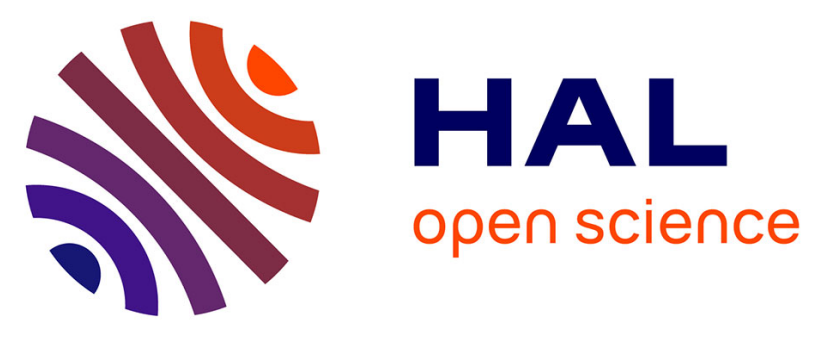

\title{
Motor chronic inflammatory demyelinating polyneuropathy ( CIDP ) in 17 patients: Clinical characteristics, electrophysiological study, and response to treatment
}

Antoine Pegat, William Boisseau, Thierry Maisonobe, Rabab Debs, Timothée Lenglet, Dimitri Psimaras, Arièle Azoulay-Cayla, Emmanuel Fournier, Karine Viala

\section{- To cite this version:}

Antoine Pegat, William Boisseau, Thierry Maisonobe, Rabab Debs, Timothée Lenglet, et al.. Motor chronic inflammatory demyelinating polyneuropathy ( CIDP ) in 17 patients: Clinical characteristics, electrophysiological study, and response to treatment. Journal of the Peripheral Nervous System, 2020, 25 (2), pp.162-170. 10.1111/jns.12380 . hal-03007177

\section{HAL Id: hal-03007177 \\ https: / hal.sorbonne-universite.fr/hal-03007177}

Submitted on 16 Nov 2020

HAL is a multi-disciplinary open access archive for the deposit and dissemination of scientific research documents, whether they are published or not. The documents may come from teaching and research institutions in France or abroad, or from public or private research centers.
L'archive ouverte pluridisciplinaire HAL, est destinée au dépôt et à la diffusion de documents scientifiques de niveau recherche, publiés ou non, émanant des établissements d'enseignement et de recherche français ou étrangers, des laboratoires publics ou privés. 


\section{Motor chronic inflammatory demyelinating polyneuropathy (CIDP) in 17}

2 patients: clinical characteristics, electrophysiological study, and response to

3 treatment

4 Antoine Pegat ${ }^{1,2}$, William Boisseau ${ }^{2}$, Thierry Maisonobe ${ }^{2,}$ Rabab Debs ${ }^{2}$, Timothée Lenglet ${ }^{2}$,

5 Dimitri Psimaras ${ }^{2,3}$, Arièle Azoulay-Cayla ${ }^{2}$, Emmanuel Fournier $^{2}$, Karine Viala $^{2}$

61 - Service de neurologie C pathologies neuromusculaires, service d'explorations fonctionnelles

7 neurologiques, Hôpital Neurologique Pierre Wertheimer, Hospices Civils de Lyon, Bron, France

82 - Département de Neurophysiologie Clinique, Hôpital Pitié Salpêtrière, Assistance publique -

9 Hôpitaux de Paris, Paris, France

103 - Service de Neurologie 2-Mazarin, Hôpital Pitié Salpêtrière, Assistance publique - Hôpitaux de

11 Paris, Paris, France et Université Pierre et Marie Curie Paris 6, Centre de Recherche de l'Institut

12 du Cerveau et de la Moelle Epinière (CRICM), UMRS 975, Paris, 75013 France, Inserm U 975,

13 CNRS, UMR 7225, Paris, 75013 France

14 Running title: Motor CIDP in 17 patients

15 Correspondance :

16 Antoine Pegat,

17 Service de neurologie C pathologies neuromusculaires, Hôpital Neurologique Pierre Wertheimer,

1859 boulevard Pinel, F-69677 Bron, France

19 Tel : +33(0)4 72357607 Fax : +33(0)4 27855196

20 Email : antoine.pegat@ @chu-lyon.fr 
Abstract :

22 Background and Aims: Motor chronic inflammatory demyelinating polyneuropathy (CIDP) is a 23 rare poorly described subtype of CIDP. We aimed to study the clinical and electrophysiological 24 characteristics and as response to treatment.

25 Methods: From a prospective database of CIDP patients, we included patients with definite or 26 probable CIDP with motor signs and without sensory signs/symptoms at diagnosis. Patients were 27 considered to have pure motor CIDP (PM-CIDP) if sensory conductions were normal or to have 28 motor predominant CIDP (MPred-CIDP) if $\geq 2$ sensory nerve action potential amplitude were 29 abnormal.

30 Results: Among the 700 patients with CIDP, 17 (2\%) were included (PM-CIDP n=7, MPred31 CIDP $n=10) ; 71 \%$ were male, median age at onset was 48 years (range: $13-76$ years), $47 \%$ had an 32 associated inflammatory or infectious disease or neoplasia. At the more severe disease stage, $94 \%$

33 of patients had upper and lower limb weakness, with distal and proximal weakness in 4 limbs for $56 \%$ of them. Three-quarters $(75 \%)$ responded to intravenous immunoglobulins (IVIg) and $4 / 5$ 35 patients to corticosteroids including $3 / 3$ patients with MPred-CIDP. The most frequent 36 conduction abnormalities were conduction blocks (CB, 82\%) and F-wave abnormalities (88\%).

37 During follow-up, 4/10 MPred-CIDP patients developed mild sensory symptoms; none with PM38 CIDP did so. Patients with PM-CIDP had poorer outcome (median ONLS:4, range:2-5) 39 compared to MPred-CIDP (2, range:0-4;p=0.03) at last follow-up.

40 Conclusions: The present study found a progressive clinical course in the majority of patients 41 with motor CIDP as well as frequent associated diseases, CB, and F-wave abnormalities. 42 Corticosteroids might be considered as a therapeutic option in resistant IVIg patients with 43 MPred-CIDP. 
44 Key words: motor CIDP; pure motor CIDP; motor predominant CIDP; corticosteroid; cancer 


\section{Introduction}

47 Chronic inflammatory demyelinating polyneuropathy (CIDP) is a treatable autoimmune

48 acquired neuropathy with heterogeneous presentation. ${ }^{1,2}$ Motor CIDP is one form of atypical

49 CIDP which is described by the joint task force of the EFNS/PNS. ${ }^{3}$ However, the clinical

50 characteristics, electrophysiological features, and response to treatment of patients with motor

51 CIDP have been poorly reported given the rarity of this CIDP subtype (only 1-10\% of patients

52 with CIDP). ${ }^{4-12}$ An early age at onset is probably more frequent compared to other types of CIDP

$53 \quad 5,9$ and clinical course can be progressive or relapsing-remitting. ${ }^{5,7,9,10}$ Electrophysiological

54 studies show frequent conduction blocks (CB). ${ }^{5,7,9,10}$ Finally, intravenous immunoglobulins

55 (IVIg) treatment should be the first choice and corticosteroids have been shown to potentially

56 lead to deterioration. ${ }^{3,5,9,10}$ However, the definition of motor CIDP is not precise according to the

57 EFNS/PNS 2010 guidelines and it remains unclear whether diagnosis of motor CIDP should only

58 be based on clinical findings or whether the presence of normal sensory nerve conduction is

59 required. ${ }^{11}$

60 The aims of the present study were therefore to describe the clinical and

61 electrophysiological characteristics, as well as the response to treatment of a cohort of patients

62 with motor CIDP. 


\section{Materials and methods}

\section{Patients}

66 Data were extracted from a prospective single center (Pitié-Salpêtrière hospital, Paris, France)

67 database which includes all consecutive patients with CIDP between January $1^{\text {st }} 2008$ and

68 October $31^{\text {st }}$ 2018. Detailed material and methods have been reported elsewhere. ${ }^{13}$

69 Patients with definite or probable CIDP according to the EFNS/PNS 2010 guidelines, ${ }^{3}$ having a 70 motor CIDP with motor signs (weakness, cramps, fasciculations, motor cranial nerve palsy), in a

71 symmetric or asymmetric polyneuropathic distribution ${ }^{7}$ with no sensory symptoms or signs at 72 diagnosis (including normal light touch, pinprick, temperature, vibratory sensations, normal gait,

73 and negative Romberg sign), were included. Patients with focal (involvement of the brachial or 74 lumbosacral plexus or of one or more peripheral nerves in one upper or lower limb), multifocal 75 acquired demyelinating sensory and motor neuropathy (MADSAM)/Lewis-Sumner syndrome, ${ }^{3}$ 76 or multifocal motor neuropathy (MMN) ${ }^{14}$ were excluded.

77 Patients with motor CIDP were classified into 2 groups according to sensory explorations upon 78 electrodiagnostic examination (EDX): patients with normal sensory conduction studies were 79 classified as having pure motor CIDP (PM-CIDP). Patients with at least 2 sensory nerves with 80 sensory nerve action potential (SNAP) amplitude under the lower limit of normal value of the 81 EDX laboratory were classified as having motor predominant CIDP (MPred-CIDP). 


\section{Data collection}

86 Disease course and response to treatment as well as laboratory workup and nerve biopsy were

87 retrospectively collected from medical files. Antiganglioside antibodies including anti-GM1, -

88 GM2, -GM3, -GD1a, -GD1b, -GT1b and GQ1b antibodies of both IgM and IgG isotypes were

89 detected by using immunodot assay.

90 Asymmetrical weakness was defined by a difference of 1 medical research council (MRC) grade

91 if strength was $\mathrm{MRC}>3$, and 2 MRC grades if strength was $\mathrm{MRC} \leq 3$, as is used in MMN. ${ }^{14}$

92 The course of the disease was classified as either progressive or relapsing-remitting (defined as 2

93 episodes with intervening remission and relapse unrelated to a change in treatment). A positive

94 response to treatment was defined as an improvement by at least 2 MRC grades in any muscle

$95 \mathrm{and} /$ or by 1 point improvement in the overall neuropathy limitations scale (ONLS). A treatment-

96 dependent patient was one who needed regular administration of his/her treatment to maintain its

97 benefit and to avoid the risk of rapid relapse.

\section{Neurophysiological investigations}

100 EDX was performed - as previously described - ${ }^{15}$ by an experienced electromyographer using a

101 Viking Nicolet electromyograph. Demyelinating features (including definite and probable partial

102 motor conduction block, CB) on motor nerves were defined according to the EFNS/PNS 2010

103 guidelines. ${ }^{3}$ The first EDX performed at the Pitié-Salpêtrière Hospital for each patient was

104 considered in the present study. An inversed sensory ratio was defined by a median/sural or

105 ulnar/sural or radial/sural SNAP amplitude ratio <1, without median or ulnar nerves with

106 entrapment. Somatosensory evoked potentials (SSEP) were performed for certain patients. 
107 Proximal conduction was suggestive of demyelination if it showed abnormal conduction in the 108 N8-N22 segment or N18-N22 conduction time. ${ }^{16}$

\section{Statistical analyses}

111 Continuous variables were compared using Student's test or Wilcoxon rank sum test, and discrete 112 variables using Chi-squared test or Fisher's exact test. The level of significance was set at $\mathrm{p}<0.05$.

113 All calculations were performed using the statistical software $\mathrm{R}^{*}$ ( $\mathrm{R}$ core team, 2017).

\section{Ethics statement}

116 Clinical data were obtained in accordance with ethical standards laid down in the 1964

117 Declaration of Helsinki and its later amendments. All patients were informed and gave their 118 consent. For this retrospective study, no authorization from an ethics committee was required. 


\section{Results}

121 Among 700 patients with CIDP in the database, 17 (prevalence of $2 \%$ ) were included in the 122 present study: 7 had PM-CIDP and 10 MPred-CIDP. All but 1 patient were classified as having 123 definite CIDP (Patient \#3 with PM-CIDP had probable CIDP; Table 1).

\section{Clinical features}

125 There were 5 women and 12 men (Table 1). The median age at onset was 48 years (range: 13-76 126 years), 6/17 patients (35\%) were less than 30 years old at onset. In most patients, onset was

127 chronic (12/16 patients [75\%]). The initial symptom was lower limb (LL) weakness in 10/16 128 patients $(59 \%$, Table 1$)$. At the more severe disease stage, the majority of patients had 4-limb 129 weakness (15/16 patients [94\%], Table 1). The median ONLS at the more severe disease stage 130 was 4 points (range: 3 -10) and 3 patients used a single crutch (patient \#2, \#12, and \#13). Median 131 ONLS at last follow-up was 3 (range: 0-5); 4 patients had a ONLS score of 0 or 1 . An associated 132 disease was found in 8/17 patients (47\%), including 4/17 patients (24\%) presenting with cancer 133 (Table 1). In 2 patients (patient \#7 and \#8), cancer was diagnosed during CIDP investigations 134 (B-cell lymphoma, and epidermoid carcinoma of lung) and in 2 others, it was diagnosed 1 year 135 (patient \#11 with palate cancer) and 11 years (patient \#5 with generalized cancer) after CIDP 136 diagnosis.

\section{Disease course and response to treatment}

138 The median follow-up duration was 4 years (47 months, range: 1-153 months). During follow-up,

1392 patients (patients \#5 and \#11) died due to cancer complications and 1 was lost to follow-up 140 (patient \#8). Clinical course was progressive in most patients (12/17 patients; 71\%) and 141 relapsing-remitting in $5 / 17$ patients (29\%, Table 2). Spontaneous improvement occurred at the 
142 beginning of disease in 3/17 patients (18\%; patient \#6, \#14 and \#17). Among those concerned, 143 12/16 patients (75\%) had a positive response to IVIg, 4/5 (80\%) to corticosteroids (including 3/3 144 with MPred-CIDP and 1/2 with PM-CIDP; the patient with PM-CIDP who did not respond to 145 corticosteroids did not show clinical deterioration), and 2/5 (40\%) to plasma exchange (PE; 146 patient \#7 had a lymphoma treatment (splenectomy) associated with PE, Table 2). Only 4/13

147 patients $(31 \%)$ experienced successful treatment discontinuation with a median duration of 12 148 months (range: 7-42, Table 2).

\section{Paraclinical exams}

150 Neurophysiological investigations

151 In 11/17 patients (65\%), EDX was performed before the onset of immunomodulatory treatment.

152 Among all 17 patients, 15 (88\%) had definite CIDP (Table 3). The median interval between first 153 clinical signs and EDX was 24 months (range: 3-72 months). The median number of motor 154 nerves tested was 8 (range: 5-8) and sensory nerves tested was 8 (range: 5-10).

155 The 2 most frequent demyelinating features on motor nerves were $\mathrm{F}$ wave abnormalities 156 (prolongation or absence) and CB; F wave abnormalities were found in 15/17 patients (88\%) 157 representing 53/100 nerves (53\%) and multifocal CB in 14/17 patients (82\%) representing 58/95 158 nerves $(61 \%$, Table 3$)$. The median number of CB per patient was 4 (range: 0-8). In total, 34/64 $159 \mathrm{CB}$ were definite (53\%) and 30/64 were probable (47\%). Among the $64 \mathrm{CB}, 26(41 \%)$ were 160 located in the ulnar nerve (Table 3). In the 37 nerves without $\mathrm{CB}, 21$ had abnormal $\mathrm{F}$ waves 161 (57\%). Prolonged DL and reduced CV were rare and found in 3/17 (18\%) and 6/17 (35\%) 162 patients respectively (Table 3, mostly on nerves with CB). 
163 The median number of reduced distal compound muscle action potential (CMAP) amplitude per 164 patient was 4 (range: 0-8). Fibrillation potentials or positive sharp waves at rest were found in $16511 / 17$ patients (65\%). A total of 5/17 (29\%) patients had fasciculations in 1 muscle, and 1 patient 166 (patient \#11) had fasciculations in 4 muscles (Table 3).

167 Among patients with MPred-CIDP, the median number of abnormal SNAP amplitude was 4.5 168 (range: $2-9), 9 / 10$ patients $(90 \%)$ had an inversed sensory ratio.

169 Five patients (all were MPred-CIDP) had data for SSEP and 3/5 (60\%) had proximal conduction 170 abnormality suggestive of demyelination.

171 Other paraclinical exams

172 Two patients (patients \#11 and \#14,) had IgM anti-GM1 antibodies (patient \#11 had associated 173 anti-GQ1b antibodies) and 1 patient had IgM anti-GM2 antibodies (patients \# 4, Table 1). The 174 mean CSF protein concentration was $73 \mathrm{mg} / \mathrm{dl}$ (range: $0.36-150 \mathrm{mg} / \mathrm{dl}$ ) and CSF protein was 175 mildly elevated (>50 mg/dl) in 11/14 patients (79\%; Table 1$)$. Nerve biopsy was performed in 3 176 patients (all had MPred-CIDP) and found absence of inflammatory infiltrate in 3 patients and

177 evidence of demyelination or remyelination in 2 patients.

\section{Comparison of PM-CIDP and MPred-CIDP patients}

179 During follow-up, 4/10 (40\%) patients with MPred-CIDP developed mild sensory symptoms, 180 none of those with PM-CIDP did so $(\mathrm{p}<0.001$, Table 4$)$. These sensory symptoms consisted in 181 tingling in extremities which appereared with a median delay of 18 months (range: 8-216 182 months), but none had gait or limb ataxia nor hypoesthesia (patients \#14 and \#16 : tingling in 4 183 extremities, patients \#15 and \#17: tingling limited to hands). Median follow-up duration was 184 similar for patients with PM-CIDP (47 months, range: 1-81) and patients with MPred-CIDP (44 
185 months, range: 3-153, Table 4). The median follow-up of the 4 patients with mild sensory 186 symptoms was 57 months (range: 39-153). The median ONLS at last follow-up in patients with 187 PM-CIDP was 4 (range: 2-5) compared to 2 (range: 0-4) for those with MPred-CIDP (p = 0.03); 1884 patients with MPred-CIDP had an ONLS of 0 or 1 while none of those with PM-CIDP did so 189 (Table 4). Concerning the response to treatments, response to IVIg was not significantly different 190 between PM-CIDP and MPred-CIDP patients ( $\mathrm{p}=1$, Table 4). Among the patients treated by 191 corticosteroids, 1/2 PM-CIDP patients responded compared to 3/3 MPred-CIDP patients ( $\mathrm{p}=0.4$; 192 Table 4). 


\section{Discussion}

The present study shows that motor forms of CIDP can be considered as variants of CIDP that present with specific clinical, electrophysiological, and response to treatment courses. More precisely, the present study confirms that motor CIDP seem to be (i) rare; ${ }^{4-12}$ (ii) tend to occur more often in males; ${ }^{5,8,10}$ (iii) at a young age at onset; ${ }^{5,9}$ (iv) present with frequent and multifocal $\mathrm{CB}^{5,7,9,10}$ and $\mathrm{F}$ wave abnormalities ${ }^{5,7,10}$ on EDX; and (v) respond well to IVIg. ${ }^{5,7,9,10}$

Other potential features of motor CIDP are highlighted herein. First, a high proportion of patients (almost half of them) presented with associated diseases. Three patients were diagnosed with cancer, either during CIDP investigations or in the first year of evolution. It is difficult to conclude on a possible link between cancer and motor CIDP given that tumors were diverse and not necessarily known to be associated with paraneoplastic neurologic disorders. Demyelinating neuropathy with predominantly motor form has been reported in association with lymphoma. ${ }^{17}$ Melanoma, lung or esophageal cancer, have also been described in motor CIDP. ${ }^{18,19}$ In a previous cohort of CIDP patients (not only motor CIDP), our group reported a concurrent illness in only one quarter of patients. ${ }^{13}$ Therefore, thorough investigations aiming at identifying diseases associated with motor CIDP are required. Second, in agreement with two previous studies, ${ }^{5,7}$ a majority of patients herein experienced a progressive clinical course. Conversely, all patients from the studies by Kimura et al. ${ }^{10}$ and Sabatelli et al. ${ }^{9}$ presented with a relapsingremitting course. Finally, despite the use of various immunomodulatory treatments, the majority of patients experienced a poor clinical outcome: only a small quarter of patient had an acceptable ONLS score at 0 or 1 at the end of follow-up. This is discordant with the results from Kimura $e t$ al. who reported that patients with motor CIDP experienced very good outcome, better than patients with other CIDP types. ${ }^{10}$ 
218 almost all nerves in which abnormal CV and DL were found indicates that $\mathrm{CB}$ are the cause of 219 these demyelinating parameters and therefore should not be considered alone. Furthermore, in 220 this context and with an objective of diagnosis, it is of note that the ulnar nerve was the most 221 affected by $\mathrm{CB}$, but also that $\mathrm{CB}$ can be present only in proximal segments. Conversely to $\mathrm{CV}$ 222 and DL, F wave abnormalities were found both in nerves with and without CB, which should also 223 be kept in mind during diagnostic workup.

No consensual definition is available concerning the diagnosis of pure motor CIDP. The 225 EFNS/PNS guidelines, which provide a more precise definition of motor CIDP, are currently 226 under review. In the present study a clinical definition of motor CIDP, at diagnosis, was used and 227 patients were included independently of sensory conduction abnormality upon EDX. Thus, 2 228 groups were identified herein, patients without sensitive nerve abnormalities (PM-CIDP) and 229 those with (MPred-CIDP), as assessed by EDX. There was no significant difference in terms of 230 clinical and paraclinical features between the 2 groups, except for outcome (patients with PM231 CIDP had poorer outcome), and the development of sensory symptoms during follow-up (all 232 patients who developed subtle sensory signs had MPred-CIDP). Some of the MPred-CIDP 233 patients who developed mild sensory symptoms did not however develop ataxia or hypoesthesia 234 as in common CIDP, and remained motor predominant. It is possible that these patients have an 235 intermediate and milder form, which lies on the spectrum between the motor and typical forms. 236 Donnedu et al. had previously reported the possibility that atypical CIDP, including motor CIDP, 237 evolved towards typical CIDP. ${ }^{7}$ The higher proportion of patients (more than half of patients) 238 moving towards a typical form of CIDP observed by the authors could be explained in part by the 239 longer median follow-up (6 years as opposed to 4 years herein). ${ }^{7}$ Taken together, for a patient 
240 with a motor form, the existence of a sensory involvement upon EDX might be a predictive factor

241 for moving towards a typical CIDP. In a similar way, patients with other atypical CIDP forms

242 (pure sensory CIDP, MADSAM/Lewis-Sumner, distal acquired demyelinating symmetric

243 neuropathy [DADS]) can evolve into typical CIDP. ${ }^{7,15,20}$

244 Finally, pure motor CIDP has been frequently reported as a steroid unresponsive

245 pathology. 5,9,10,21 In the present study however, the majority of patients who underwent

246 corticosteroid treatment showed improvements, but these concerned mainly patients with

247 MPred-CIDP group. This result is in line with the study by Doneddu et al. which reported a

248 beneficial effect of corticosteroids in 3/7 patients, but exclusively in patients with MPred-CIDP. ${ }^{7}$

249 Using these recent data, corticosteroids might be considered as a therapeutic option in resistant

250 IVIg motor CIDP patients, at least for patients with abnormal sensory conduction. More data are

251 needed to rule on the efficiency of corticosteroid in PM-CIDP patients.

252

The current body of data indicates that 2 main alternative diagnoses of neuropathy could

253 be considered in the context of motor deficits: MMN and motor neuron disease. Motor CIDP and

254 MMN share several features: pure motor involvement, young age at onset, good response to IVIg

255 therapy, possible antiganglioside antibodies, and multiple CB on EDX. However, they differ on 3

256 major aspects: motor CIDP is associated with diffuse weakness, more diffuse

257 electrophysiological damage, and a possible improvement using corticosteroids. However, some

258 MMN patients may develop a more diffuse distal and proximal weakness later in the disease

259 course indicating that a continuum may exist between MMN and motor CIDP. The association of

260 pure motor deficit, amyotrophy, and sometimes diffuse cramps and fasciculation (as in patient

261 \#11), suggests motor neuron disease. Motor neuron disease and motor CIDP can share common 
262 EDX abnormalities, for instance, reduced distal CMAP, fibrillation, fasciculations, and CB; the

263 latter are reported transitory in patients with rapidly progressive motor neuron disease. ${ }^{22-24}$

The present study has several limitations inherent to its retrospective observational design 265 and the fact that it is based on a small number of patients because of the rarity of the disease.

266 Furthermore, due to our expert center status, the first EDX was performed after 267 immunomodulatory treatment initiation in a third of patients which could affect results. Finally, 268 after initial investigations and treatment introduction, some patients were redirected to their local 269 center which explains a shorter follow-up period.

270 To conclude, beyond the previously reported features of motor CIDP including its low 271 prevalence, good response to IVIg, and frequent $\mathrm{CB}$ and F wave abnormalities upon EDX, the 272 present study found a progressive clinical course in the majority of patients and frequent 273 associated diseases. In contrast to MPred-CIDP patients, PM-CIDP patients seem to have poorer 274 outcome and did not develop sensory symptoms during follow-up. Corticosteroids might be 275 considered as a therapeutic option in resistant IVIg patients with MPred-CIDP but more data are 276 needed to rule on the efficiency of corticosteroids in PM-CIDP patients.

279 Acknowledgments: We thank Philip Robinson and Véréna Landel (DRCI, Hospices Civils de 280 Lyon) for help in manuscript preparation. 


\section{Reference}

1. Vallat J-M, Sommer C, Magy L. Chronic inflammatory demyelinating polyradiculoneuropathy: diagnostic and therapeutic challenges for a treatable condition. The Lancet Neurology. 2010 Apr;9(4):402-12.

2. Rabin M, Mutlu G, Stojkovic $\mathrm{T}$ et al. Chronic inflammatory demyelinating polyradiculoneuropathy: search for factors associated with treatment dependence or successful withdrawal. Journal of Neurology, Neurosurgery \& Psychiatry. 2014 Aug $1 ; 85(8): 901-6$.

3. Van den Bergh PYK, Hadden RDM, Bouche P, et al. European Federation of Neurological Societies/Peripheral Nerve Society Guideline on management of chronic

4. Gorson KC, Allam G, Ropper AH. Chronic inflammatory demyelinating polyneuropathy: clinical features and response to treatment in 67 consecutive patients with and without a monoclonal gammopathy. Neurology. 1997 Feb;48(2):321-8.

5. Busby M, Donaghy M. Chronic dysimmune neuropathy. Journal of Neurology. 2003 Jun 1;250(6):714-24.

6. Mahdi-Rogers M, Hughes RAC. Epidemiology of chronic inflammatory neuropathies in southeast England. European Journal of Neurology. 2014 Jan;21(1):28-33. 
progression and treatment response. Data from the Italian CIDP Database. Journal of 304 Neurology, Neurosurgery \& Psychiatry. 2019 Feb;90(2):125-32.

8. Ikeda S, Koike H, Nishi R et al. Clinicopathological characteristics of subtypes of Psychiatry. 2019 Jun 21;

9. Sabatelli M, Madia F, Mignogna T, Lippi G, Quaranta L, Tonali P. Pure motor chronic inflammatory demyelinating polyneuropathy. J Neurol. 2001 Sep;248(9):772-7.

10. Kimura A, Sakurai T, Koumura A et al. Motor-dominant chronic inflammatory demyelinating polyneuropathy. Journal of Neurology. 2010 Apr;257(4):621-9.

11. Nobile-Orazio E. Chronic inflammatory demyelinating polyradiculoneuropathy and variants: where we are and where we should go. J Peripher Nerv Syst. 2014 Mar;19(1):2-13.

12. Querol L, Rojas-Garcia R, Casasnovas C, et al. Long-term outcome in chronic inflammatory demyelinating polyneuropathy patients treated with intravenous immunoglobulin: A retrospective study: Long-Term Effectiveness of IVIg in CIDP. Muscle \& Nerve. 2013 Dec;48(6):870-6

13. Viala K, Maisonobe T, Stojkovic $\mathrm{T}$ et al. A current view of the diagnosis, clinical variants, response to treatment and prognosis of chronic inflammatory demyelinating polyradiculoneuropathy. Journal of the Peripheral Nervous System. 2010 Mar;15(1):506.

14. Joint Task Force of the EFNS and the PNS. European Federation of Neurological 
Societies/Peripheral Nerve Society Guideline on management of multifocal motor neuropathy. Report of a Joint Task Force of the European Federation of Neurological Societies and the Peripheral Nerve Society - first revis. Journal of the Peripheral Nervous System. 2010 Dec;15(4):295-301.

15. Viala K. Follow-up study and response to treatment in 23 patients with Lewis-Sumner syndrome. Brain. 2004 Aug 2;127(9):2010-7.

16. Koutlidis RM, Ayrignac X, Pradat P-F et al. Segmental somatosensory-evoked potentials as a diagnostic tool in chronic inflammatory demyelinating polyneuropathies, and other sensory neuropathies. Neurophysiol Clin. 2014 Sep;44(3):267-80.

17. Viala K, Behin A, Maisonobe T, et al. Neuropathy in lymphoma: a relationship between

19. Mostoufizadeh S, Souri M, de Seze J. A Case of Paraneoplastic Demyelinating Motor Polyneuropathy. Case Reports in Neurology. 2012;4(1):71-6.

20. van Dijk GW, Notermans NC, Franssen H, Wokke JH. Development of weakness in patients with chronic inflammatory demyelinating polyneuropathy and only sensory symptoms at presentation: a long-term follow-up study. J Neurol. 1999 Dec;246(12):1134-9. 
345 21. Molenaar DS, van Doorn PA, Vermeulen M. Pulsed high dose dexamethasone treatment 346 in chronic inflammatory demyelinating polyneuropathy: a pilot study. Journal of 347 Neurology, Neurosurgery \& Psychiatry. 1997 Apr 1;62(4):388-90.

348 22. Echaniz-Laguna A, Degos B, Mohr M, Kessler R, Urban-Kraemer E, Tranchant C. A 349 study of three patients with amyotrophic lateral sclerosis and a polyneuropathy 350 resembling CIDP. Muscle \& Nerve. 2006 Mar;33(3):356-62.

351 23. Rajabally YA, Jacob S. Chronic inflammatory demyelinating polyneuropathy-like 352 disorder associated with amyotrophic lateral sclerosis. Muscle \& Nerve. 2008 $353 \quad$ Jul;38(1):855-60.

354 24. Ahdab R, Créange A, Saint-Val C, Farhat W-H, Lefaucheur J-P. Rapidly progressive 355 amyotrophic lateral sclerosis initially masquerading as a demyelinating neuropathy. Neurophysiologie Clinique/Clinical Neurophysiology. 2013 Jun;43(3):181-7. 


\section{Tables}

Table 1: Clinical and laboratory characteristics

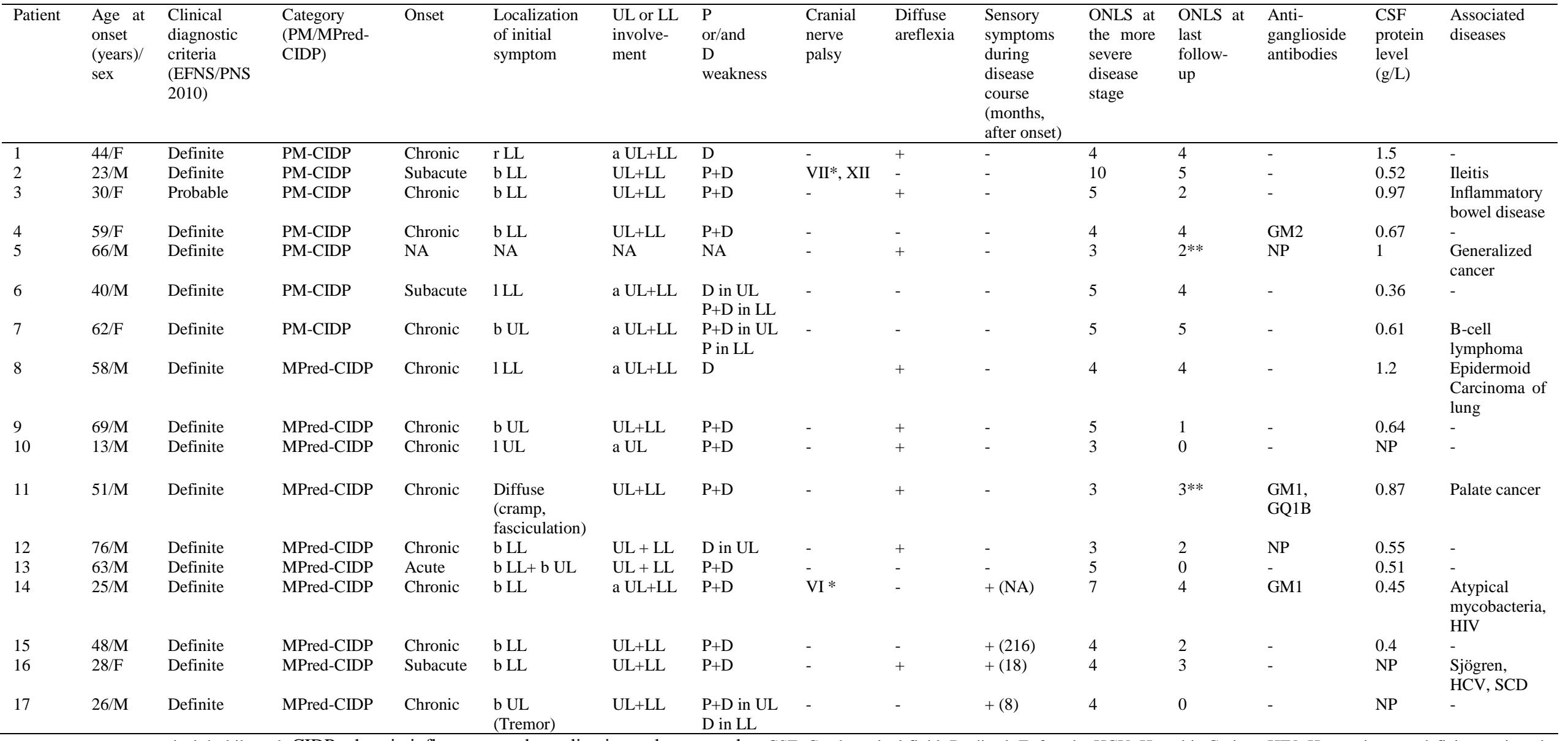

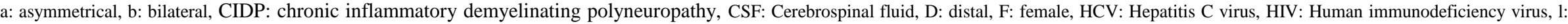

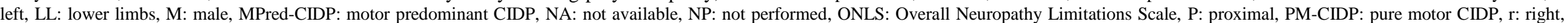
SCD: Sickle cell disease, UL: upper limbs, *: bilateral, +: present, -: absent, **: at last follow-up before the death 
Table 2: Disease course and response to treatment

\begin{tabular}{|c|c|c|c|c|c|c|c|c|c|}
\hline Patient & $\begin{array}{l}\text { Category } \\
\text { (PM/MPred- } \\
\text { CIDP) }\end{array}$ & $\begin{array}{l}\text { Duration of } \\
\text { follow-up } \\
\text { (months) }\end{array}$ & $\begin{array}{l}\text { Disease } \\
\text { course }\end{array}$ & $\begin{array}{l}\text { Response } \\
\text { to IVIg }\end{array}$ & $\begin{array}{l}\text { Response } \\
\text { to CS }\end{array}$ & $\begin{array}{l}\text { Response } \\
\text { to PE }\end{array}$ & $\begin{array}{l}\text { Other IS } \\
\text { treatments }\end{array}$ & $\begin{array}{l}\text { Successful } \\
\text { discontinuation } \\
\text { of IVIg, CS, or } \\
\text { PE without } \\
\text { relapse } \\
\text { (month \#) }\end{array}$ & $\begin{array}{l}\text { Current } \\
\text { therapy }\end{array}$ \\
\hline 1 & PM-CIDP & 81 & Progressive & + & NT & - & MM & - & IVIg+MM \\
\hline 2 & PM-CIDP & 56 & Progressive & + & - & NT & MM & $+(42)$ & 0 \\
\hline 3 & PM-CIDP & 12 & Progressive & + & NT & NT & NT & - & IVIg \\
\hline 5 & PM-CIDP & 47 & Progressive & - & + & + & $\mathrm{Az}$ & - & $\mathrm{PE}^{*}$ \\
\hline 6 & PM-CIDP & 60 & RR & + & NT & NT & NT & - & IVIg \\
\hline 7 & PM-CIDP & 16 & Progressive & - & NT & + & NT & NT & $\mathrm{PE}$ \\
\hline 8 & MPred-CIDP & 3 & Progressive & NT & NT & NT & NT & NT & NT \\
\hline 9 & MPred-CIDP & 36 & Progressive & + & + & - & MM & $+(12)$ & MM \\
\hline 10 & MPred-CIDP & 56 & Progressive & - & + & - & NT & - & CS+IVIg \\
\hline 11 & MPred-CIDP & 120 & Progressive & + & NT & NT & NT & - & IVIg* \\
\hline 16 & MPred-CIDP & 39 & $\mathrm{RR}$ & + & NT & NT & Rituximab & $+(7)$ & 0 \\
\hline 17 & MPred-CIDP & 65 & $\mathrm{RR}$ & + & NT & NT & NT & $+(12)$ & 0 \\
\hline
\end{tabular}

Az: azathioprine, CIDP: chronic inflammatory demyelinating polyneuropathy, CS: corticosteroids, IS: immunosuppressive, IVIg: intravenous immunoglobulins, MM: mycophenolate mofetil, MPred-CIDP: motor predominant CIDP, NT: not tried, PE: plasma exchange, PM-CIDP: pure motor CIDP, RR: relapsing-remitting, +: yes, -: no, *: last follow up before death, \#: months since stopping treatment without relapse 
Table 3: Electroneuromyography data

\begin{tabular}{|c|c|c|c|c|c|c|c|c|c|c|c|c|c|c|}
\hline \multirow[b]{2}{*}{ Patient } & \multirow[b]{2}{*}{$\begin{array}{l}\text { Category } \\
\text { (PM/MPred- } \\
\text { CIDP) }\end{array}$} & \multirow[b]{2}{*}{$\begin{array}{l}\text { Electro- } \\
\text { diagnostic } \\
\text { criteria } \\
(*, \\
\text { months) }\end{array}$} & \multicolumn{4}{|c|}{ Demyelinating features on motor nerves } & \multicolumn{4}{|c|}{ Topography of CB } & \multirow[b]{2}{*}{$\begin{array}{l}\text { Number of } \\
\text { motor } \\
\text { nerve with } \\
\text { abnormal } \\
\text { CMAP } \\
\text { amplitude }\end{array}$} & \multirow[b]{2}{*}{$\begin{array}{l}\text { Number of } \\
\text { sensory } \\
\text { nerves } \\
\text { with } \\
\text { abnormal } \\
\text { SNAP } \\
\text { amplitude }\end{array}$} & \multirow[b]{2}{*}{$\begin{array}{l}\text { Fibrillation } \\
\text { or PSW at } \\
\text { rest }\end{array}$} & \multirow[b]{2}{*}{$\begin{array}{l}\text { Fascicu- } \\
\text { lation at } \\
\text { rest }\end{array}$} \\
\hline & & & $\begin{array}{l}\text { Prolonged } \\
\text { DL }\end{array}$ & $\begin{array}{l}\text { Reduced } \\
\mathrm{CV}\end{array}$ & $\begin{array}{l}\text { Abnormal } \\
\text { F waves }\end{array}$ & $\begin{array}{l}\text { Number } \\
\text { of } \mathrm{CB}\end{array}$ & Median & Ulnar & $\begin{array}{l}\text { Com- } \\
\text { mon } \\
\text { fibular }\end{array}$ & Tibial & & & & \\
\hline 1 & PM-CIDP & Definite (48) & + & - & - & 5 & 2 (D) & $3(2 \mathrm{D}, \mathrm{I})$ & & & 7 & 0 & + & - \\
\hline 2 & PM-CIDP & Definite (7) & - & - & + & 0 & & & & & 8 & 0 & + & - \\
\hline 3 & PM-CIDP & Possible (3) & - & - & + & 3 & $1(\mathrm{D})$ & & $2(\mathrm{D})$ & & 4 & 0 & + & - \\
\hline 4 & PM-CIDP & Definite (5) & + & - & + & 8 & 1 (D) & $3(2 \mathrm{D}, \mathrm{I})$ & 2 (D) & 2 (D) & 8 & 0 & + & - \\
\hline 5 & PM-CIDP & Definite (72) & - & - & + & 0 & & & & & 0 & 0 & - & - \\
\hline 6 & PM-CIDP & Definite (60) & - & + & + & 3 & & $2(\mathrm{I})$ & 1 (D) & & 2 & 0 & + & + \\
\hline 7 & PM-CIDP & Definite (36) & - & - & + & 3 & & 2 (D) & 1 (D) & & 4 & 0 & + & - \\
\hline 8 & MPred-CIDP & Definite (3) & - & + & + & 4 & 2 (D) & 2 (D) & & & 6 & 9 & + & + \\
\hline 9 & MPred-CIDP & Definite (36) & - & + & + & 0 & & & & & 3 & 6 & - & - \\
\hline 10 & MPred-CIDP & Definite (72) & - & + & + & 6 & $4(2 \mathrm{D}, 2 \mathrm{I})$ & 2 (D) & & & 4 & 2 & - & + \\
\hline 11 & MPred-CIDP & Definite (4) & - & - & + & 7 & $2(\mathrm{I}, \mathrm{P})$ & $3(\mathrm{I}, 2 \mathrm{P})$ & 2 (D) & & 4 & 5 & + & + \\
\hline 12 & MPred-CIDP & Definite (12) & + & - & + & 4 & 1 (D) & 2 (D) & 1 (D) & & 8 & 7 & + & - \\
\hline 13 & MPred-CIDP & Definite (6) & - & + & + & 6 & 2 (D) & $3(\mathrm{D}, 2 \mathrm{P})$ & 1 (D) & & 2 & 4 & - & - \\
\hline 14 & MPred-CIDP & Probable (24) & - & - & - & 3 & & $1(\mathrm{I})$ & 2 (D) & & 1 & 3 & - & - \\
\hline 15 & MPred-CIDP & Definite (30) & - & - & + & 4 & & & 2 (D) & 2 (D) & 3 & 2 & + & + \\
\hline 16 & MPred-CIDP & Definite (24) & - & - & + & 1 & 1 (D) & & & & 8 & 5 & + & - \\
\hline 17 & MPred-CIDP & Definite (14) & - & + & + & 7 & 2 (D) & $3(2 \mathrm{D}, \mathrm{I})$ & 2 (D) & & 2 & 3 & - & - \\
\hline
\end{tabular}

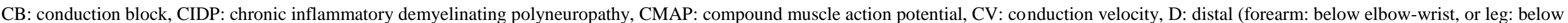

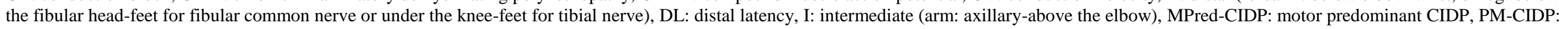
pure motor CIDP, P: Proximal (Erb'point-axillary), PSW: Positive sharp wave, SNAP: sensory nerve action potential, *: delay between first clinical signs and EDX, +: present, - : absent 
Table 4: Characteristics of patients with PM-CIDP and MPred-CIDP

\begin{tabular}{llll}
\hline & PM-CIDP & MPred-CIDP & p value \\
\hline Sex male/female & $3 / 4$ & $9 / 1$ & 0.1 \\
Age at onset, years, median (range) & $44(23-66)$ & $49.5(13-76)$ & 1.0 \\
Progressive onset & $4(67 \%)$ & $8(80 \%)$ & 0.6 \\
Development of sensory symptoms & $0(0 \%)$ & $4(40 \%)$ & 0.1 \\
& & & \\
Disease course and response to treatment & & & \\
Duration of follow-up, months, median (range) & $47(1-81)$ & $44(3-153)$ & 0.7 \\
RR disease course & $1(14 \%)$ & $4(40 \%)$ & 0.3 \\
Response to IVIg & $5 / 7(71 \%)$ & $7 / 9(78 \%)$ & 1 \\
Response to CS & $1 / 2(50 \%)$ & $3 / 3(100 \%)$ & 0.4 \\
ONLS at the more severe disease stage (range) & $5(3-10)$ & $4(3-7)$ & 0.29 \\
ONLS at last follow-up (range) & $4(2-5)$ & $2(0-4)$ & $0.03 *$ \\
& & & \\
Electroneuromyography & & & \\
Abnormal F waves & $6(86 \%)$ & $9(90 \%)$ & 1.0 \\
Number of CB, median (range) & $3(3-8)$ & $4(0-7)$ & 0.3 \\
Prolonged DL & $2(29 \%)$ & $1(10 \%)$ & 0.54 \\
Reduced CV & $1(14 \%)$ & $5(50 \%)$ & 0.3 \\
Abnormal CMAP amplitude, median (range) & $4(0-8)$ & $3.5(1-8)$ & 0.6 \\
Abnormal SNAP amplitude, median (range) & $0(0-0)$ & $4.5(2-9)$ & $<0.001^{*}$ \\
Fibrillation or positive sharp wave at rest & $6(86 \%)$ & $5(50 \%)$ & 0.3 \\
Fasciculation at rest & $1(14 \%)$ & $4(40 \%)$ & 0.3 \\
\hline
\end{tabular}

CB: conduction block, CIDP: chronic inflammatory demyelinating polyneuropathy, CMAP: compound muscle action potential, CS: corticosteroids, CV: conduction velocity, DL: distal latency, IVIg: intravenous immunoglobulin, MPred-CIDP: motor predominant CIDP, ONLS: overall neuropathy limitations scale, PM-CIDP: pure motor CIDP, RR: relapsing-remitting, SNAP: sensory nerve action potential, $*$ : $\mathrm{p}<0.05$ 